\title{
Multiple drug therapy: implications for field work and training at ALERT
}

\author{
M BECX-BLEUMINK \\ All Africa Leprosy and Rehabilitation Training Centre, P.O. Box \\ 165, Addis Ababa, Ethiopia
}

\section{Introduction}

The introduction of multiple drug therapy (MDT) of limited duration is beyond doubt a breakthrough in the campaign against leprosy. However, the application of MDT is not merely the distribution of two or three drugs instead of one; it also implies an extensive re-organization and up-grading of virtually all aspects of a leprosy control programme (1).

With the introduction of MDT the principles of leprosy control, early detection of patients and effective treatment for a sufficient period of time do not change. Proper planning and organization of MDT, in order to guarantee optimal implementation as well as the development of a built-in system for evaluation, are managerial tasks par excellence. It cannot be overemphasized that it is of extreme importance that detailed guidelines for implementation of all aspects of MDT are defined before MDT is introduced.

Implications of Multiple Drug Therapy for field work

With the introduction of treatment regimens of limited duration, rapid changes can be observed in the leprosy control field situation.

In many leprosy control programmes, including the ALERT Leprosy Control Programme, MDT is implemented in area after area. Some years after the introduction of MDT, different areas will be in different phases of implementation. Four phases can be distinguished:

1. Preparatory phase

2. Initial phase of implementation

3. Intermediate phase of implementation

4. Final phase of implementation. 
Preparatory phase. The duration of this phase may vary widely and depends on factors such as the number of patients under treatment, the number of clinics, number of staff, accessibility in the area, availability of resources. During this phase main activities are:

- Selection of patients for MDT. This includes clinical and bacteriological examination of all patients under treatment, and reassessment of the classification. So that patients are not unnecessarily exposed to drugs drugs that can give side-effects, many managers of leprosy control programmes have realized that, prior to introduction of MDT, plans should be defined and executed for the release from treatment of patients who should be considered to have received sufficient chemotherapy and need not be given MDT. In Ethiopia the policy of at least 5 years of regular treatment with dapsone monotherapy for paucibacillary (PB) patients and 10 years of regular treatment for multibacillary (MB) patients, in the absence of clinical and baceriological activity at the end of that period, was defined (2).

- Changing to new recording and reporting systems.

- (Re)defining staff tasks.

- Defining and allocation of resources.

- Education of patients and communities concerning the new treatment.

During this phase the workload is high; additional resources, especially manpower and vehicles, may need to be assigned temporarily to the area under preparation for MDT.

Initial phase of Implementation of MDT. This phase lasts 6 months to 1 year and is finished at the same time as the $\mathrm{PB}$ patients will have completed their course of chemotherapy.

Main activities during this phase are:

- Start of MDT by the PB and MB patients.

- Clinical examination of the PB patients at the end of the 6 months' course of chemotherapy. 
- Release from treatment of the PB patients who have fulfilled the criteria of attendance.

- Applicatior of rules which have been defined for PB patients who did not fulfill the criteria for attendance.

- Application of instructions for follow-up examinations of $\mathrm{PB}$ patients after their release from treatment.

The workload during this phase is high; especially at the time of assessment and release from treatment of the PB patients, and may require temporary assignment of additional resources.

Intermediate phase of Implementation of Multiple Drug Therapy. This phase covers the second and third year of implementation of MDT, and lasts until the MB patients have completed their course of chemotherapy. Main activities during this phase are:

- Annual clinical and bacteriological assessment of the MB patients.

- Release from treatment of MB patients; starting two years after introduction of MDT.

- Application of rules that have been defined for MB patients who did not fulfill the criteria of attendance; starting three years after introduction of MDT.

- Application of instructions for follow-up examinations after release from treatment.

During this phase the workload gradually decreases, but at the times of assessment of the patients the workload is considerable.

Final phase of Implementation of Multiple Drug Therapy. This phase starts at the time the majority of the MB patients have been released from treatment, which is usually 3 years after the introduction of MDT. Of the patients who started MDT during the initial phase of implementation, only those who did not fulfill the criteria for skin smear results remain under MDT.

The majority of the patients under treatment will be those who have been diagnosed as having leprosy after the introduction of MDT. 
Main activities during this phase are:

- Diagnosis of new patients and relapses.

- Continuation of follow-up of patients after their release from treatment.

The workload will have decreased tremendously. In properly planned and executed MDT programmes, in areas where leprosy control activities with a good coverage of the population have been carried out prior to the introduction of MDT, the reduction of patients under chemotherapy will be in the order of 808 or more 3 years after the introduction of MDT.

This phase will last for many years and certainly until the time leprosy has ceased to be a public health problem.

Training and Evaluation. In order to guarantee proper preparation for and implementation of MDT, training of staff is extremely important. In the ALERT Leprosy Control Programme a phased system of training of staff has been introduced; the phases of implementation of MDT are discussed during separate workshops. Furthermore evaluation is a continuous process which covers the different stages of planning, programming, and execution of the activities. This concerns operational, epidemiological and performance evaluation.

The objective of leprosy control, other than the provision of chemotherapy:

An effective treatment which will interrupt transmission of the disease, cure the patients and at the same time prevent the occurrence of disabilities.

Leprosy control should, however, deal with more than providing treatment. In the excitement of implementing MDT there is a danger that the care for patients who have developed disabilities due to the disease is not given the attention it ought to have. As has been pointed out, the caseload, related to patients under chemotherapy, will gradually decrease for the first years of implementation of MDT. However, the total workload will remain high during many years, due to: 
- Provision of patient care directed at existing and potential disabilities. In many leprosy control programmes 308 or more of the known leprosy patients are in need of regular care for existing disabilities or threats of increased disability. With the enormous decrease in patients under chemotherapy an obvious approach is to give more attention to these patients. It is important that resources are not cut in response to the decrease in case load. Of equal importance is that available surgical resources for correction of disabilities can take care of patients who are in need of reconstructive surgery.

- The need for regular follow-up examinations of patients who have been released from treatment. At present neither the risk of relapse in patients who have completed MDT nor criteria for identification of patients at high risk of developing a relapse are known.

\section{The ALERT leprosy control programme}

The ALERT Leprosy Control Programme is responsible for leprosy control in Shoa Administrative Region. This region is in the centre of Ethiopia; it covers an area of about $85,000 \mathrm{~km}^{2}$, with a poulation of 8.75 million. The region is divided into one urban and eleven rural districts. Leprosy diagnostic and treatment services are provided in 292 centres; 608 of these are attached to the general medical services and 408 are leprosy clinics, which have been established in those areas where a general medical services does not yet exist (3).

\section{Multiple Drug Therapy in the ALERT Leprosy Control Programme}

MDT was introduced into the ALERT Leprosy Control Programme according to the recommendations of the World Health Organization in 1982 (4) in January 1983. Paucibacillary patients are treated for a period of 6 months; with dapsone, self-administered daily, and rifampicin, administered monthly under supervision (2). Multibacillary patients are treated for a period of at least 2 years, and until the skin smears have become negative; with dapsone and clofazimine self administered daily, and rifampicin and clofazimine administered under supervision monthly (2). 


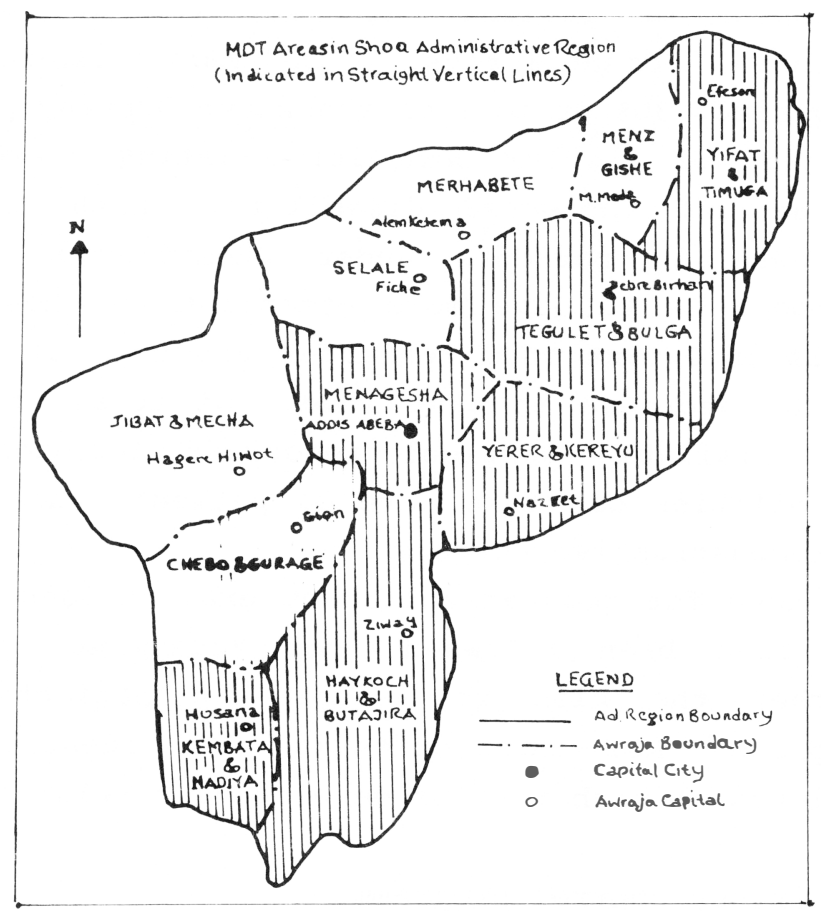

Figure: 1 MDT areas in Shoa Administrative Region

During 1983 MDT was introduced in two districts in the north-eastern part of the region, Tegulet \& Bulga and Yifat \& Timuga, known as the Debre Berhan area; including 64 clinics (Fig. 1).

During 1984 MDT was extended to three districts in the central part of the region, Addis Ababa, Menagesha and Yerer \& Kereyu, in the Addis Ababa area; including 48 clinics (Fig. 1). In December 1985, MDT was introduced in two districts in the southern part of the region, Haykoch \& Butajira and Kembaica \& Hadya, the Southern Shoa area, 61 clinics (Fig. 1). During 1986 Selale and Menz \& Gishe districts (Fig. 1) will be included in the MDT programme, while during the period 1987 to 1989 MDT will be introduced in Merhabete, Chebo \& Gurage and Jibat \& Mecha districts (Fig. 1).

At present the different phase of preparing for and implementation of MDT can be clearly distinguished:

Merhabete, Chebo \& Gurage and Jibat \& Mecha districts are in the first stage of preparation for MDT. 
- Selale and Menz \& Gishe districts are in the extensive phase of preparation for MDT.

- The districts of the Southern shoa area are in the initial phase of implementation of MDT.

- The districts of the Addis Ababa area are in the intermediate phase of implementation of MDT.

- The districts of the Debre Berhan area are in the final phase of implementation of MDT.

For the departments' managerial staff, this situation requires different approaches as regards matters such as priorities in supervision, detection of weaknesses, allocation of resources, priorities in training, evaluation of services.

Proper short term and long term planning at central level are essential in order to meet the priorities and requirements in the different areas.

Some results of implementation of MDT

During the period January 1983 to January 1986, 7,587 patients, 3,320 PB and 4,267 MB patients, have been put under MDT.

of the 2,438 PB patients who started MDT during 1983 and 1984, 2,199 patients (90.28) completed their course of treatment within a period of 9 months; 196 patients $(8.08)$ had their treatment discontinued because of irregularity of attendance, while 43 patients (1.8\%) were either transferred to a non-MDT area, (19 patients), died (12 patients) or continued the treatment after 9 months (12 patients). By January 1986 803 MB patients had been released from MDT.

In the Debre Berhan MDT area no patients had been released from treatment prior to introduction of MDT, because at that time instructions for release from treatment of patients in Ethiopia had not been defined, while, due to incomplete records, it did not appear to be possible to apply the guidelines given by the World Health Organization (5). In this area the number of patients under chemotherapy has decreased from 3,509 in January 1983 to 596 in January 1986, a reduction of 838 during a period of 3 years.

In the Addis Ababa area 1,952 patients were released from treatment prior to introduction of MDT. After March 1984, 2,744 patients were put under MDT. By January 1986 the number of patients under treatment in the area was 2,268, compared 
with 5,186 patients in July 1983; a reduction of 568 in a period of $21 / 2$ years.

In the Southern Shoa area 2,104 patients were released from treatment prior to the introduction of MDT. By January 1986, one month after the start of implementation of MDT, 1,190 patients had started their course of MDT and 1,157 patients were still under dapsone monotherapy.

\section{Prospects of Leprosy Control in the ALERT Leprosy Control}

\section{programme}

During the period July 1982 to July 1985 the number of patients under chemotherapy, MDT and dapsone monotherapy, in the Shoa region has decreased from 20,908 to 10,507; a reduction of 508 in a period of 3 years. This decrease is due to the application of instructions for release from treatment of patients after dapsone monotherapy (2) and the introduction of MDT.

By 1993, 3 years after the last district of Shoa region will have been covered by MDT, all but a few patients under chemotherapy will be newly diagnosed patients and patients who have developed a relapse of the disease, who, one hopes, will be few.

Assuming that there will be no major changes in the number of newly diagnosed patients in the region, the number of patients under chemotherapy will be in the order of 2,000 3,000 at any time from 1993 onwards. This is a reduction of 85-908 in the number of patients under anti-leprosy treatment within a period of 10 years. With 292 leprosy diagnostic and treatment centres in the region the average number of patients per clinic will then be 7 to 10 , compared with 70 in 1983 .

As has been pointed out earlier, follow-up of patients after release from treatment and the provision of care for patients with disabilities will continue to demand attention. The first group of patients will gradually decrease and with a follow-up of 5 years after release from treatment, from 1998 onwards mainly concern newly diagnosed and relapsed patients.

If the trend towards a steady, though slow decrease in the proportion of new patients who already have severe disabilities at the time of diagnosis of leprosy continues, as it has during recent years, and if reactions are diagnosed early, and properly treated and continuous care can be given to prevent 
an increase of disability, the second group of patients will gradually decrease as well. However, this decrease is not expected to take place very fast. Without being pessimistic, the number of patients who will be in need of care will by far outreach the number of patients under chemotherapy for quite a number of years.

The figure for 1985 of 188 of new patients who had already a disability grade 2 or 3 at the time of diagnosis of leprosy is still high. Our method of detection of patients has been almost exclusively passive over the years. A more active detection of patients, which is one of our priorities, whenever this is feasible, could reveal a sharper decline in the proportion of new patients with severe disabilities.

Another matter for our serious concern is the integration of leprosy and tuberculosis control activities $(3,6)$. The leprosy control infrastructure could provide a sound basis for the extension of tuberculosis control in rural areas.

The need for proper and careful evaluation of the effectiveness of MDT requires that the leprosy control infrastructure remains unchanged during the next few years.

\section{Implications of MDT for training at ALERT}

During the international training courses, training in leprosy control is provided through:

- Sessions in the classroom:

Since 1983 the number of hours assigned to leprosy control has increased from 4 to about 20. During the sessions general principles of leprosy control, planning, organization and implementation of MDT and evaluation of the leprosy control services are discussed. It is very stimulating to discuss the problems trainees experience in general, and with implementation of MDT in particular. It is sad to hear that it is not exceptional that MDT is implemented with little or no planning beforehand and without preparation of guidelines. The diversity of the trainees' experience in the field of leprosy control, and of their future responsibilities, especially the trainees attending the doctors' courses, makes it a challenging task to come up to their expectations of the course. 
- Training in the field:

During the supervisors' courses the trainees are taken into the field twice, for a period of 1 week. This gives us the opportunity to show them areas in different phases of preparation for and implementation of MDT. The trainees who attend the doctors courses are also taken into the field for 1 week. An increasing number of them stay for an additional 1 to 2 weeks' leprosy control in-service training, after the formal training course, a policy we strongly support.

At present and during the next few years trainees can be shown the different phases of implementation of MDT. However, within a period of 6 to 7 years, all districts of Shoa Region will have reached the final phase of implementation of MDT.

An increasing number of trainees will get their field training in clinics with a few patients under chemotherapy. This situation is very illustrative of the results that can be expected in case MDT is properly planned and executed. Furthermore, much of the process of implementation of MDT can be learnt from available records. However, trainees have already expressed their disappointment with attending clinics which have only a few patients under chemotherapy. Especially those on doctors' courses used to obtaining much of their experience through the examination and classification of patients, the taking of skin smears, and the filling in of patients cards and registers in the field.

In areas which are in the final phase of implementation of MDT the majority of the clinics have too few patients to give trainees sufficient experience as regards these matters.

The final phase of implementation of MDT in the Debre Berhan area has brought us into the following situation:

By January 1986 the average number of patients per clinic was 9, compared with 55 in January 1983. About 30 out of the 64 clinics in the area are at present suitable for training 
purposes. Soon these will not number more than 24 clinics. These are clinics which are accessible by car and which have five or more patients under chemotherapy. As clinics for supervised treatment are conducted at 4-weekly intervals, an average of 6 clinics in this area can be included in a 1-week field training course. This number is quite sufficient, however, since the days the clinics are conducted and the travelling distances between clinics have to be taken into consideration. The travelling distances especially are a matter of concern; it would be unrealistic and very expensive to travel hundreds of kilometres per day.

We shall soon face a comparable situation in the Addis Ababa area, while gradually the other areas will follow. A leprosy control programme has, however more areas of responsibility than the provision of chemotherapy, and these also need to be given due attention during field training courses:

- care for patients with disabilities, under field conditions.

- follow-up of patients after release from MDT, with its many operational aspects.

- practical problems of diagnosis of relapses, and reactions after release from treatment.

- case-detection activities.

When the implementation of MDT has reached its final phase, these responsibilities become relatively more important, not only for the field work, but also in the training programmes.

\section{References}

1. Becx-Bleumink, M. Planning, organization and analysis of feasibility studies on Multiple Drug Therapy of leprosy. Working paper presented at a WHO workshop on reorientation of leprosy control for English speaking countries. Banjul, 24-27 July 1984 .

2. All Africa Leprosy and Rehabilitation Training Centre Manual for implementation of Multiple Drug Therapy, 1985.

3. Becx-Bleumink, M. Annual Report of ALERT for 1985, 72-110. 
$80 \quad$ M Becx-Bleumink

4. World Health Organization. Chemotherapy of leprosy for control programmes. Report of a WHO Study Group. Technical Report Series 675, 1982 .

5. World Health Organization, A guide to leprosy control. $1980,27-28$.

6. Becx-Bleumink, M. New developments in ALERT Leprosy Control Programme and the issues of integration. Eth. J. of Health Development 1984, 2, 49-55. 